\title{
Communication and disease*
}

\author{
Ian D. Carter \\ World Health Organisation, Geneva
}

\begin{abstract}
Synopsis
Being a healthy traveller involves communication in more than one sense of the word.

Health administrations have a responsibility to prevent illness associated with international travel and trade without undue interference to these inevitable activities. Past experience of travel-associated disease transmission should determine epidemiologically justified and cost-effective preventive measures.

The aeroplane transmits disease faster but should not become the scapegoat for travel-associated ill health. Viral influenza can spread rapidly because of air travel but the latter's role in transmission of diseases such as cholera and smallpox in the past or viral haemorrhagic fevers in the future may not be clear cut.

Surveillance activities are essential to enable the earliest possible detection of disease because measures taken with the intention of keeping a disease out of a country cannot be foolproof. Frank and rapid exchange of information between national epidemiological services is essential for effective control of international spread of diseases.

National health administrations have an obligation to provide travellers with reliable information. A specific programme co-opting the medical profession, travel agencies of every form and the media is necessary.

The World Health Organisation should collaborate with national health administrations in the prevention of disease associated with travel.
\end{abstract}

One of the responsibilities of the Unit of Epidemiological Surveillance of Communicable Diseases in the Division of Communicable Diseases in the World Health Organisation (W.H.O.) is the day-to-day administration of the International Health Regulations (I.H.R.) (W.H.O. 1974). This, together with the activities inherent in the surveillance of communicable diseases at an international level, does provide an opportunity to be involved in at least some aspects of disease associated with travel. It should be stressed, however, that the views expressed here are not necessarily those of the Organisation.

The word 'communication' has many nuances but the 'actual passing from one place to another' and 'the exchange of information' are probably the two most pertinent to being a healthy traveller. An attempt is made to consider 'exchange of information' in its broadest sense and to consider the virtues of healthy travel not only for the traveller himself but for the community to which he relates. International travel and trade are facts of life and, although they are inevitably associated with ill health, it is our responsibility to reduce the amount of this ill health as much as possible. However, like a number of our health problems, although by no means all, our achievements are going to be related to how much we are prepared to pay.

It would be extremely difficult, if not impossible, to quantify meaningfully the amount of ill health which has resulted from people, animals, plants and goods of all

*Prepared in the Unit of Epidemiological Surveillance of Communicable Diseases, W.H.O., Geneva. 
sorts moving about the world. Epidemics of smallpox, cholera and plague have left an indelible mark in our recorded history (Hinman 1966). But other diseases, for example, measles when introduced into a country with an extremely low level of immunity such as Fiji in 1875 (Ashburn 1947), have decimated the populations involved. But what have we learnt and what can we learn with respect to the role of travel in the transmission of disease and, more importantly, what are the most cost-effective preventive measures? One hopes that we shall soon be entering an era in which costeffectiveness rather than emotion will determine the level of compromise to be made in order to have the maximum security against the international spread of disease with a minimum interference with world traffic.

\section{The role of air travel}

One wonders at times if the aeroplane is not the scapegoat for the ill health that one associates with international travel. It is often proposed that, because an ever increasing number of people are flying faster and faster to and from more and more places, there is a greater risk of spreading disease. For the rapidity of spread of, for example, a new strain of influenza virus or sexually transmitted diseases, air travel must plead guilty. Beta-lactamase-producing strains of Neisseria gonorrhoeae were first reported in early 1976 but by October 1977 the isolation of 97 cases of gonorrhoea due to this strain had been reported from 13 other countries (Weekly Epidemiological Record (W.E.R.) 1977). On the other hand one must not lose sight of the fact that perhaps the most lethal of influenza epidemics spread throughout the world in 1918-19 (Burnet and Clark 1942).

We often stress that a traveller can now go by air from the remotest part of the world to any other within the incubation period of any disease. But it is perhaps also fair to suggest that in a high percentage of cases the potentially sick traveller may reach the security of his home, or a place where he will receive adequate medical care, before he becomes sick or a source of infection. There is not a documented incident of anybody contracting smallpox on a plane. There was one man who travelled by plane from Australia to Scandinavia and who developed smallpox after his arrival, but the source of his infection was never identified (W.E.R. 1963). Cholera-infected food has been served to air travellers, but there has been a minimum of secondary spread when those concerned have arrived in countries with an acceptable level of environmental sanitation (W.E.R. 1973). The effect of serving staphylococcal-contaminated ham and mayonnaise contaminated with Salmonella typhimurium in 1975 and 1976 on flights into European capitals had far more dramatic and serious consequences (W.E.R. 1976).

Cholera is, however, of great interest and concern in considering the role of air travel in the spread of disease. In the present pandemic, one cannot deny the possible involvement of air travel, for example at some phase in the westward movement of the disease from Indonesia which began in 1960. But the ten years that it took to reach Africa is not a reflection of speed and volume of air travel (W.E.R. 1974). And why is it that South America has been spared until now? One should emphasize the 'until now' because there is no way to prevent the introduction of cholera into any country.

In fact there must be very few diseases whose introduction can be prevented. If a 
disease is introduced in spite of the precautions that are taken, a certain expense is involved in controlling the situation. Thus, although we may be able to influence the frequency of introduction, this represents a different situation which must take into consideration the cost-effectiveness of the procedures we employ to keep the disease out and to control it when it is inevitably introduced.

One could briefly consider in a similar way some of the other diseases which have traditionally been held in awe. Louse-borne typhus is very closely linked to social conditions and for many years has rarely been associated with international travel. Plague, which merits intensive surveillance wherever it occurs, is now only reported from a few focal areas which are outside the main streams of travel (W.H.O. 1978). Effective treatment has reduced its killing force which was so terrifying in the past. Apart from the importation of one human case into the U.S.A. in 1966, one has to go back to the 1920 s to find documented evidence of the introduction of this disease (W.H.O. 1978). In recent years there have been instances of tourists contracting yellow fever in Africa (W.E.R. 1979), and this is of vital importance and warrants specific consideration. But there has been no evidence for several decades that yellow fever has been spread as a result of international traffic.

\section{Effectiveness of measures to prevent introduction of disease}

It is possible, therefore, that air travel per se is not responsible for any significant quantitative difference in the spread of diseases internationally even though that spread may occur more quickly. It could be said that this reflects the effectiveness of the quarantine measures that have been in existence over the last 20 or 30 years. (It is appreciated that these may not have been the only specific preventive measures that were implemented.) We now know however that some of them, such as the requirement of a vaccination certificate, had no effect in preventing the introduction of cholera into any country.

The situation with respect to smallpox is more complex. For example, there was no recorded introduction of smallpox into Australia after 1938 (W.H.O. 1980). (If there had been an unobserved introduction, the likelihood of limited secondary transmission would have been high. because the level of immunity in the general population was probably relatively low.) Can it be said that this absence of introduction was due to the effectiveness of the quarantine service? The difficulty is to reconcile this experience with that of other countries. For example, over the same period there were at least 20 introductions into the United Kingdom (W.H.O. 1980), and it would be extremely difficult to identify any essential difference in the operation of quarantine services in the two countries. People leaving either country were in general well vaccinated; this was important because a study of introductions has shown that, in the majority of instances, it was travellers who had not been properly vaccinated who introduced the disease on returning to their own country. But, in addition, there was a regular flow of travellers from Asian countries towards the United Kingdom, which was not the case for Australia. It is interesting just briefly to link the experience of Switzerland, where it can be noted that there was one introduction both in 1962 and in 1963 (W.H.O. 1980). However, in that country, although there was a statutory requirement for smallpox vaccination, the visual control of certificates could not be compared with that of the 
United Kingdom and Australia. It is not intended here in any way to cast doubt on the value of either the vaccination process or any quarantine service, but only to suggest that it is difficult to assess objectively the role that they played in preventing the introduction of disease.

The only real value in attempting to assess the effectiveness of the measures that have been taken in the past to prevent the introduction of disease is to determine what we should be doing now and what we should plan to do in the future on the basis of what we believe we know now. It is by attempting to assess the true role that the aeroplane has played in the spread of diseases in the past that we can try to foresee objectively its importance now and in the future for other diseases such as the viral haemorrhagic fevers. We perhaps need to be careful that we do not allow the relative ease with which the air-traveller can be controlled, in comparison with one arriving by any other means of transport, to influence unduly the belief in the value of the measures taken at airports by health administrations. It has been sad, from time to time, to see countries, while insisting on cholera vaccination certificates from all travellers, suddenly forced to realize that an epidemic situation has developed within their own borders.

\section{Movement of vectors}

Before considering in detail the possible approaches that could be adopted to prevent, or rather reduce as much as possible, the spread of disease by the human traveller, mention should be made of things other than man that travel and contribute to ill health in humans. Apart from insect vectors of human disease, which at some time or other and at some stage of their development must have been found in every form of transport known to man, there are animals, birds, fish, reptiles and the whole range of food products which could be implicated. However, it is not possible, here, to deal with the potentially nefarious effects of these usually unwanted travellers, particularly as an enormous economic interest with regard to crops and livestock is concerned and hence the agricultural and veterinary disciplines are involved.

The introduction of Anopheles gambiae into Brazil some 50 years ago (Duffy 1977, p. 201-233), into Egypt 40 years ago (Duffy 1977, p. 234-254), and of a number of species of Anopheles into Guam in the 1970s are well known (Ward et al. 1976). There have been indigenous cases of malaria in people living close to airports in Europe (W.E.R. 1978), where the most logical if not only explanation is that transmission was the result of infected mosquitoes arriving in aircraft. Without detracting from the importance of these events, we should perhaps ask ourselves why this has not happened more often: it possibly has, and some malaria cases, for example, could well have passed undetected.

The programmes leading to the eradication of Anopheles gambiae from Brazil and Egypt were outstanding achievements, and in the light of difficulties associated with some vector control activities in more recent times are even more impressive. Is it possible that the ecological balance was in favour of eradicating the introduced species even though the introduction may have been of relatively long standing? This role of nature could account to some extent for the comparatively few instances in which accidental introduction of insect vectors of human disease have led to dramatic outbreaks. For example, in the Pacific there seems to be a natural boundary, on one side of which one finds Anopheles and on the other they do not exist. Now this line must 
have been crossed an infinite number of times by planes or boats before, during and after the Second World War, and it is difficult to imagine that the insect in one form or another did not have the opportunity to make that journey. But apart from Guam these introductions do not appear to have been maintained. Many entomologists will agree that the spraying procedures, as they have been practised over recent years, could not have been expected to prevent the introduction of insect vectors. When one considers the hardiness of the eggs of Aedes aegypti and the traffic on and above the Tasman Sea, it is difficult to explain why that species has not become a permanent resident in New Zealand.

\section{The risk of disease introduction in perspective}

It would seem therefore that we are faced with a dilemma, or perhaps a series of dilemmas. One can argue theoretically that one or other form of travel could be responsible for extremely serious communicable disease situations. For example, the introduction of yellow fever (either by an infected mosquito or a human in the incubation period) into Asia and elsewhere is a long-standing fear, while some of the 'newer' viral haemorrhidgic fevers have been of great concern in recent years. The fact that our worst fears have not been realized does not seem to dispel this fear nor allow us to look at the potential dangers more critically.

In other situations we have seen the feared event occur even though the so-called protective measures have been taken. The introduction of cholera despite the requirement of a certificate from every traveller has been referred to above. Let us consider the situation if a case of yellow fever did get into a developing country in Asia. Is there any reason to believe that we would not see the same build-up of cases as has been the experience with cholera in many countries? There are situations where strict and comprehensive airport insect control measures have been taken, but relatively frequent introductions of exogenous insects have still occurred. Another perplexing situation is the apparent lack of appreciation of the specificity of risk. Is it acceptable that travellers can go into rural western Africa and contract yellow fever, while certificates are rigidly demanded from travellers coming from Nairobi, for example, where there has never been a proven human case? Many of the present so-called preventive measures, which presumably can be very expensive in terms of personal resources, cannot be justified epidemiologically and cannot be guaranteed to exclude the introduction of disease. We have seen cases of viral haemorrhagic fever in travellers detected after they have arrived in a country, and there is no easy way to determine the small number of cases. if any, that go undetected.

\section{An epidemiological service}

In other words, we must accept that, irrespective of the efficacy of any mechanism to keep a disease out of a country, there must be a mechanism to determine as early as possible that cases of that disease are occurring within the country. This is easy to say but far more difficult to do than generally appreciated, as it presupposes a functioning, effective epidemiological service. By this is meant a service which can: 
(a) perform effective disease surveillance including the collection, collation, analysis and reporting of information;

(b) investigate disease outbreaks rapidly and competently;

(c) develop and apply appropriate control and preventive measures as indicated by disease investigations;

(d) make recommendations concerning ongoing and new control and preventive programmes based upon surveillance data;

(e) develop the necessary information to be able to categorize diseases by their impact on the quality of life from the stand-point of economics, health hazards, morbidity and mortality;

(f) contribute to discussions concerning cost-effective interventions for diseases of national importance;

(g) assist in developing priorities for the country's public health (prevention) programmes;

(h) develop evaluation techniques for the above programmes;

(i) assist in teaching biostatistics, epidemiology and surveillance to personnel of the medical and allied professions and to paramedical personnel.

This list of activities may not be exhaustive, nor may it be in the order which represents the needs of an individual country. More importantly, the form in which such a service is established may vary considerably from one country to another. It will be different, for example, in a country with independent states, or in a socialist country, or where a good network of laboratories exists or does not exist as the case may be.

\section{Disease surveillance}

It is fitting that this symposium should be held in Edinburgh, so close to the Communicable Diseases (Scotland) Unit at Ruchill, Glasgow. where effective disease surveillance was first established in the United Kingdom in 1969 (Health Bulletin 1970). It was stressed at the time the Unit was set up that all the elements for good surveillance existed and all that was necessary was to bring these elements together. Similarly, the creation of the Communicable Disease Surveillance Centre in Colindale in 1977 extended the epidemiological functions of the Public Health Laboratory Service beyond epidemiological research to include responsibility for communicable disease surveillance and control (W.E.R. 1980).

With effective surveillance activities as an integral part of the national epidemiological service, a national health administration is in a strong position to evaluate objectively the measures that are being practised at the points of entry to a country. When this is done, it becomes very clear that there is no hard and fast set of procedures that can necessarily be transferred from one country to another, nor even from one part of a country to another. It may, for example, be epidemiologically justifiable to control an aircraft for insect vectors of human disease (or animal and plant disease under the direction of the appropriate department) coming from a specific airport and landing in an area of a country where the climatic conditions are such that the vector could be expected to survive.

The way an individual country sample surveys its incoming or outgoing food 
products depends on a large number of criteria; the nature of the foodstuff (fresh or processed), the manner of shipment, and the existing agreements with the manufacturer and/or the national health administration of the country with which the trade exists, are just a few of the aspects to be considered. The United Kingdom, along with a number of other countries, has already modified the way in which the health clearance of ships is managed. In the same manner, the epidemiological service can propose the most cost-effective approach to each potential health risk associated with international travel.

It is probable that the main brunt of the burden will fall on the surveillance activities which themselves must be continually evaluated to ensure that they keep abreast of the changing epidemiological situation. Because the surveillance activities of the national epidemiological service are already concerned with the early detection of a change in the health situation within the country (be it an outbreak of measles in an unvaccinated group of the population or the appearance of illness of the nervous system in workers associated with the introduction of a new factory process), the detection of disease originating outside the country is a logical additional responsibility. In other words, the good surveillance system brings to light any travel-associated disease along with any other disease, and the related epidemiological investigation assigns an appropriate significance to the "travel aspect" including, if necessary, the proposal of specific preventive measures either within the country, at its border or beyond in collaboration with another national health administration.

To what extent, however, do countries have functioning, effective epidemiological services with reliable disease surveillance? Many developed countries do have good population, environmental, mortality and morbidity data. There are investigations of single cases and of epidemics, and there are sound epidemiological, clinical and serological surveys based on reliable laboratory findings. But the process of bringing these elements together, so that the most effective action can be taken with a minimum of delay, is lacking or not fully developed.

If some developed countries do not have effective surveillance activities, despite the necessary elements being available, is it realistic to believe that they can exist in developing countries? It is not uncommon for the health administration of a developing country to justify the requirement of cholera vaccination certificates from all travellers, or some other barrier type approach, on the inability to afford surveillance activities. One could argue that the investment represented in the health training of quarantine staff at ports and airports could be used to far greater advantage in the development of surveillance activities. But in practice this may not be so simple. The personnel of quarantine services have often been in place for many years and are resistant to change. It may be extremely difficult to recycle them in the present prolonged period of unemployment.

There is also an important political aspect which in the majority of instances cannot be dissociated from the media. (This also relates to a facet of communication which is subsequently taken up in greater detail.) Despite the fact that attempts by regulatory and quarantine measures to prevent the introduction into non-infected countries of diseases coming from infected areas have failed, completely or partially, and will continue to fail, it is apparently difficult for the media to accept that the solution at the present time is an effective surveillance programme. It is understandable therefore that the politician defers to public protest stimulated by the media. which may be 
misguided, and maintains procedures which are expensive but of little or no effect merely to show that 'something is being done'

\section{Surveillance at the periphery}

Is it to be assumed, therefore, that developing countries are to be denied surveillance programmes? On the contrary, it is not a question of whether they can afford to have surveillance activities but whether they can afford not to have them. In passing it should be mentioned that the impetus of the W.H.O.-sponsored Expanded Programme on Immunization and the Diarrhoeal Diseases Control Programme in the developing countries is growing, and that these constitute two vital elements of the Organisation's overall commitment to primary health care and to the goal of Health for All by the Year 2000. These two programmes demand the development of effective surveillance of the diseases concerned, which in turn can only stem from effective national epidemiological services. As suggested earlier, however, the manner in which these services are developed is determined by the conditions in a given country. In Singapore, for example, excellent communicable disease surveillance activities are carried out within the Ministry of the Environment.

The challenge is going to be an individual one for each national health administration that is truly committed to communicable disease control activities. How may the limited personnel and financial resources that are available be used both to know that something is happening in the villages at the periphery, where the sick people are, and to do something about it? Some of the elements, and perhaps in a large number of countries many or most of the elements that have been mentioned above as being required for good surveillance, are simply not available. Initially, all that may be possible is to establish, within individual villages, a norm for a limited number of conditions, perhaps based only on symptoms. For example, for a given village of a certain population, it will be determined that up to 10 cases of fever or 15 cases of diarrhoea are 'acceptable'. Beyond that figure somebody in the village will take a certain action: it may be to walk to the next village, or to go by boat to the nearest health centre or to stop a mail truck which passes twice a week on a road 20 kilometres away. Initially, it may not even involve a written message, but it will be the signal on the basis of which something must happen. This presupposes of course that the people of the village do assume responsibility for keeping themselves healthy, and that when the signal is given something does happen. Without that community involvement and without the assurance that there will be a response, be it a visit from a teacher, a health aid, a nurse, a doctor or somebody who will do something, then truly there will no surveillance, and there will be no primary health care worthy of the name.

\section{Exchange of information}

This basic concept of the generation of information for action leads us to the second interpretation of 'communication' in relation to travel and disease viz 'exchange of information'. It is intended here to consider communication between the health 
administrations of different countries on the one hand, and between health administrations and travellers on the other.

When there is, within one country, a communicable disease situation of interest to other countries (and it does not have to be one of the big 'stars' like plague or cholera or viral haemorrhagic fever; the recent appearance of dengue type 4 virus in Central America (W.E.R. 1982) and outbreaks of acute haemorrhagic conjunctivitis in a number of countries (W.E.R. 1981), and the ever-recurring problem of gastrointestinal disease on cruise ships (W.E.R. 1975) are classic examples), it is the quality of the epidemiological information which can be provided about the specific disease situation that ultimately determines how rapidly the incident can be controlled both economically and in terms of human illness. It is how quickly the problem is identified and the appropriate control measures implemented which is vital, and this reflects the calibre of the national epidemiological service. This approach applies to the full range of communicable diseases and not only to those on a particular list at a given time.

It is only when we have greater confidence in the ability to detect a disease situation and control it that we can expect to have true cooperation through open and rapid interchange of information. There is a paradox, however, with respect to the whole question of the provision of communicable disease information. The underlying problem is the failure to differentiate between data which evolve from a routine information system and data generated for surveillance purposes per se. Obviously a good surveillance system is going to make use of, or at least vet, communicable disease information from any source, including that resulting from routine reporting. But essentially it is looking for information for action 'now', rather than for information which can only tell us what has happened.

It has been proposed above that in some countries it may well be necessary to establish surveillance mechanisms which are not based on written reports. If we take a village situation in which there is an increase in the incidence of gastro-enteritis, this may be faithfully recorded in the routine reports of the nearest health centre, be passed up the line through district and province, and may only belatedly, or perhaps never, give rise to an epidemiological investigation. If that same village were co-operating in a surveillance system based on a norm for gastro-enteritis and which specified a certain action once the accepted number of cases had been exceeded, it is to be hoped that the epidemiological investigation which would follow - perhaps only a verbal report would identify a recently contaminated water supply, a new source of a food product or even only confirm, in the absence of a specific cause, that the outbreak was not continuing.

In other words, there may be a danger in aiming for too much in the way of routine reporting. Does it really help to know how many cases of tetanus or poliomyelitis may have occurred in an area if there is no control programme worthy of the name for these diseases in that area? On the other hand, if there is a meaningful control programme, then every single case of those two diseases is of vital importance as it reflects a defect at some stage of the programme.

The routine recording of information can be very expensive particularly in terms of time. Surveillance based on relatively few, specific, well-planned surveys may be far more cost-effective than attempting to use large amounts of data emanating from routine procedures. Surveillance then, in one sense, could be considered the ultimate 
evaluation of a communicable disease control programme. If a good surveillance system cannot detect cases the control programme must be effective. It does not imply that the method of control is the best available or that it is the most economical. These are different aspects requiring specific evaluation in the same way that a surveillance system itself must be subject to evaluation.

There is no intention here to imply that communicable disease information should not be recorded or disseminated but there is a need for greater consideration of the real value and purpose to which the information will be put. This is of particular importance for the developing country where personnel and financial resources are so limited.

In the same way that the epidemiological service within a given country should evaluate the nature of the communicable disease information available, or which needs to be generated to establish effective surveillance, it is worthwhile to consider what sort of information is really of value for dissemination between countries. Again, it is extremely difficult to generalize, particularly as individual national health administrations do not attach the same importance to the different communicable diseases. It also depends on the degree of contact both geographically and in terms of trade and traffic between a country in which the disease condition exists and the country receiving the information.

Another aspect is whether the information received is to be fed into an effective surveillance mechanism or to be used to advise potential travellers or for a combination of both purposes. If country ' $A$ ' has an effective surveillance system any information received is acted upon in relation to its epidemiological significance. Thus the report of 200 cases of poliomyelitis in country ' $\mathrm{B}$ ', where there is no control programme, may arouse no interest. The occurrence of two cases, however, in country ' $\mathrm{C}$ ', when reported with an epidemiological description suggesting an unusual segment of the population at risk, may stimulate an investigation of the level of immunity of similar population groups in country 'A'.

In the same way, therefore, that it is the quality rather than the quantity of communicable disease information fed to the central point of a national epidemiological service which is important, it is the local significance of the presence of disease which is most useful to the epidemiological service of another country. If descriptions of communicable disease situations - and what has been done about them - constitute the bulk of reports from the periphery to the centre of a national epidemiological service, that country probably has very little to fear from the occasional inevitable introduction of disease from the outside. This is not to say that good routine reporting of cases and deaths, which may take weeks and months to reach the centre of a national epidemiological service, do not have a role to play.

A similar comparison could be made with respect to the nature of the information made available from one national epidemiological service to another. It is probably of limited value to know that a certain number of cases of typhoid, for example, have occurred in a particular country, even if the locality is specified. It may, however, be extremely valuable to know that a particular canned food product which is available in a number of countries has been identified as the source. The same reasoning probably applies to most, if not all, communicable diseases. There is no reason to feel secure against the introduction of one of the viral haemorrhagic fevers just because there have been no reports of large outbreaks from those geographical areas where the disease has 
been most frequently identified. On the other hand, there is no epidemiological justification to impose measures restricting the free flow of hundreds, if not thousands, of passengers just because cases are occurring in an area where the disease is known to be endemic. In both situations, the best, if not the only, defence is effective surveillance activities. It cannot be expected that any such mechanism will be foolproof, and as an example one could suggest that every late diagnosis of a case of malaria indicates that the system is not as good as it could be. This should not be a reason for discouragement but taken as an incentive to intensify our efforts.

\section{The role of the media in communication}

Reference has been made above to the role of the media, and it is perhaps appropriate to refer here in greater detail to some of the communication aspects involved. The importance given to the detection of communicable disease, introduced or not, within a country can vary enormously depending on its consideration by the media, and unfortunately does not necessarily reflect its true epidemiological significance. The early detection of cases of cholera or viral haemorrhagic fever, for example, is a positive reflection of the existing surveillance mechanism, and the national health administration has a right to be proud to be able to make such notifications. Unfortunately, media reports tend to ignore this aspect and concentrate only on the existence of cases, sometimes even suggesting blame and demanding to know what is going to be done. If the cases have occurred in another country, the media may magnify the degree of risk of introduction (rarely accepting that limited introduction is to be expected nor assisting the health administration to put this to the public in perspective) and/or unduly emphasize the risk to potential travellers to the country reporting the disease.

The media have the right to be informed, but it is felt there is a need for much closer collaboration to enable a far greater objectivity in the presentation of communicable disease information to the public.

\section{Excessive measures}

Another and sometimes related problem is the wide range of excessive measures introduced by some national health administrations, not only in response to a notification of a communicable disease situation, but as a result of rumours which may or may not be well founded. It is here that the W.H.O. has an important role to play. There is already excellent collaboration with the majority of national health administrations with respect to the interchange of communicable disease information, but still greater understanding can be achieved. The introduction of excessive measures, either trade restrictions or unnecessary requirements for travellers, is the principal barrier to prompt and frank reporting, and unfortunately it is very often the very countries that do not report that tend to be the first to introduce such measures.

Another understandable cause for non-reporting is the fear of losing tourist trade. Paradoxically, a failure to report disease often has a worse effect as the traveller prefers to be told that a disease exists and how he can protect himself. If the traveller is in doubt 
whether a disease is present or not, particularly if the media give undue prominence to the situation, the tendency not to travel is greater. The sooner the W.H.O. is made officially aware of a communicable disease situation, the easier it is to prevent the introduction of excessive measures or have them withdrawn, and for the flow of tourists to be maintained with a minimum of interference.

One of the greatest difficulties with respect to excessive measures is the reluctance of the national health administrations concerned to assess objectively the epidemiological justification and the cost-effectiveness of the procedures employed. There can also be an element of false security associated with such procedures. It has been seen with cholera, and it applies to some other diseases.

In some countries, for example, the incidence of poliomyelitis has been maintained at a very low level for some years as result of effective vaccination programmes. A similar situation may well apply to measles in a growing number of countries in the future. The challenge in these situations is to maintain the high level of immunity on which the absence of cases depends. In the absence of overt disease, it is sometimes very difficult to motivate people to maintain the protective procedure which has produced the absence of disease. It is very tempting, therefore, to argue that, by ensuring travellers do not reintroduce the disease, the problem can be reduced. But we cannot prevent the introduction of such diseases, and, once the level of immunity has been allowed to fall, we are wilfully exposing the unvaccinated to possible serious infection. The person carrying polio or measles virus from the outside into an adequately vaccinated community does not constitute a risk, and in fact provides confirmation of the existing high level of immunity. It would seem that any available resources would be more profitably utilized in maintaining that immunity than in employing staff at points of entry into a country to look at certificates in the belief that the introduction of disease is being prevented.

One of the important lessons of smallpox eradication is that, if you want to maintain a disease-free state which has been achieved in your own country, by preventing the reintroduction of that disease, go out and collaborate with your neighbour in order to bring about the same disease-free situation in his country. One could argue that such an approach may be far more effective than is generally appreciated when applied, for example, to the introduction of vectors. If an objective study has shown that specific flights from certain airports are prone to carry insects, a collaborative effort at the source of the problem may well be both more effective and more economical. In a similar way, although acceptably much more difficult, if developed countries with good surveillance activities wish to have better communicable disease data from other countries to feed into their own system, it may be worth collaborating in the development and improvement of other national epidemiological services in general and surveillance activities in particular.

\section{Quality of information}

Although the prompt and frank interchange of information is our continual goal, we must accept that the degree of achievement is going to be a reflection of the extent of development of effective national epidemiological services and the competence of the related surveillance activities. The quality of the information made available in the 
Automatic Telex Reply Service and the Weekly Epidemiological Record is dependent on the information that these services provide. There has been a growing number of countries sending epidemiological reports to the Unit of Epidemiological Surveillance of Communicable Diseases, and an increasing proportion of these contain narrative in addition to numerical data. Not only are these reports of greater value as a feed-back to national staff, and hence an encouragement of surveillance and communicable disease control activities, but they lead to the promotion of the interchange of information between national epidemiological services. This, in turn, results in greater confidence and closer collaboration between these services, and is the only sure foundation on which to build a meaningful international surveillance programme.

\section{The traveller}

The traveller himself is a vital factor in the communication chain. In the same way that a community must be committed to maintaining its state of health, the traveller must participate in and, in many respects, accept responsibility for, the decisions taken with regard to health and the travel to be undertaken. To do this, the traveller must have access to a readily available source of reliable information. This presupposes that national health administrations have a specific programme aimed at informing the public and which involves the medical profession, travel agencies of every form and, of prime importance, the media.

A major difficulty is that the traveller needs two types of information, one which could be considered very general and the other which may need to be very specific.

We all need to appreciate that the change in our immediate environment that travel involves, irrespective of distance, may constitute a risk. Sometimes to travel a very short distance and eat food over which we have no control may be a greater hazard than to travel 10000 kilometres and stay with friends or relatives for a long period. Eating away from home in itself constitutes an increased risk and the traveller should have a basic set of gastronomical rules and know when these need to be applied and with what degree of severity. One should not really need to travel in order to find that one is possibly no longer immune to diphtheria, tetanus and poliomyelitis. One certainly should not travel extensively without such immunity, as the incidence of these diseases may well be appreciably higher than in one's usual place of residence.

Finally, one's basic general condition must be an initial consideration in any proposal to travel. Severe chronic conditions may not be a barrier to extensive travel whereas a tendency to a particular illness could become a serious concern in a strange environment.

There are other obvious aspects of general advice relating for example to the stress of travelling, change in climatic conditions and perhaps a temporary change in life-style, such as different drinking habits and an exposure to sexually transmitted diseases. It is, however, in the provision of more specific advice that difficulties arise. When should the traveller have immune serum globulin, typhoid, yellow fever, cholera or other vaccine? Even to prescribe malaria prophylaxis is not as simple as the average traveller believes it should be. The risk can be very site-specific, but unfortunately, very often neither the traveller, his travel agent nor his medical adviser can relate, with any precision, the possible risks to a given area. This often leads to what the traveller 
considers to be conflicting advice, especially if the person giving counsel wants to be sure of covering all eventualities. The picture is further complicated if a country has an obligatory health requirement for which there is no epidemiological justification.

Thus the communication of this information to the potential traveller is difficult and a serious challenge to each national health administration. The assistance which W.H.O. can provide in this respect is sometimes limited. The vaccination requirements of individual countries, as notified to the W.H.O. do not always, for example, reflect the situation at points of entry. In some situations clarification of reports related to the occurrence of communicable disease in areas of interest to travellers is difficult to obtain.

In 1981 the scope of the booklet on Vaccination Certificate Requirements for International Travel was increased to provide more general health advice for travellers. The booklet (W.H.O. 1982), which is not intended as a substitute for more comprehensive publications on this subject, is addressed to health administrations which have the responsibility to advise travellers. It is only the national health administration that can determine the most appropriate way to co-opt the aid of the medical profession, tourist agencies, shipping companies, airline operators and other bodies. A lot has been done in the form of films, posters, pamphlets, etc. in the language or languages appropriate to the country concerned, but it has to be accepted that this is a never-ending process calling for perseverance and ingenuity and backed by a realistic budget.

The issue of World Health for April 1982 looks at some of the health hazards associated with travelling and suggests some of the simple precautions that travellers can take before, during and after their journey; it also includes a brochure entitled $\mathrm{Be} a$ healthy traveller.

In their own interest, and in the interest of the community in which they live and work, travellers have an ongoing and vital responsibility on ret urning from their travel. They must, in the event of any illness, draw the attention of their medical adviser specifically to the fact that they have been travelling. Doctors in turn, of course, must be fully receptive to the patient's reference to travel.

National health administrations also have an important role in this respect, as doctors must be trained to consider that their patient may have travelled in the recent past and may be suffering from a disease that they rarely encounter. Training in this context is not meant to imply a brief reference to the problem in the basic medical course, even though it should be included there. It is intended to take into account that, during their professional life, doctors may have a varying likelihood of contact with persons who travel and need to be reminded of the potential situation. If clinicians adopt some form of algorithmic approach (W.H.O. 1978) to the signs and symptoms of patients who have travelled, it may help to ensure a rapid and accurate diagnosis without the unnecesary overreaction that quite often occurs. It is here again that we see the importance of the surveillance activities of the national epidemiological service. The information relating to an ill person who has been travelling is an essential element in the mosaic of communicable disease surveillance within any country: a surveillance that will enable the most appropriate action with a minimum of delay.

As suggested above, this rapid and free interchange of information must be extended to the international scene by the close collaboration of national epidemiological services. It is believed that W.H.O. has a role to play in this collaboration and in the 
development of an epidemiologically justified and uniform approach to the management of communicable disease situations including those associated with travel.

\section{References}

Ashburn, P. M. 1947. The Ranks of Death, p. 90. New York: Coward-McCam.

Burnet, F. M. and Clark, E. 1942. Influenza: survey of the last 50 years in the light of modern work on the viruses of epidemic influenza. Walter and Eliza Hall Institute of Research in Pathology and Medicine, Monograph No. 4. Melbourne: MacMillan.

Duffy, J. (Ed.) 1977. Ventures in World Health: The Memoirs of Fred Lowe Soper. P.A.H.O. Scientific Publication 355. Washington, D.C.: Pan American Health Organisation.

Health Bulletin 1970. The Medical Officer of Health in the control of the epidemiological communicable disease-approach. Hlth Bull. 28, No. 2, 24-25.

Hinman, E. H. 1966. World Eradication of Infectious Diseases, pp. 3-9. Springfield, Ill.: Thomas.

Ward, R. A., Jordan, B., Gillogly, A. R. and Harrison, F. J. 1976. Anopheles litoralis King and A. barbirostris group on the island of Guam. Mosquito News 36, No. 1, 99-100.

W.E.R. 1963. 38, 263.

W.E.R. 1973. 48, 297.

W.E.R. 1974. 49, 229.

W.E.R. 1975. 50, 231-232.

W.E.R. 1976. 51, 117-118.

W.E.R. 1977. 52, 357-359.

W.E.R. 1978. 53, 337.

W.E.R. 1979. 54, 368.

W.E.R. 1980. 55, 41-47.

W.E.R. 1981. 56, 293-294.

W.E.R. 1982. 57, 78-79.

W.H.O. 1974. International Health Regulations, 2nd annotated edition. Geneva: W.H.O.

W.H.O. 1978. Chronicle 32, 441 .

W.H.O. 1980. The Global Eradication of Smallpox. Final Report of the Global Commission for the Certification of Smallpox Eradication. Geneva: W.H.O.

W.H.O. 1982. Vaccination Certificate Requirements for International Travel and Health Advice to Travellers. Geneva: W.H.O. 